\title{
Impact of Chess Learning on Processing Speed of School-Going Children
}

\author{
Veena Easvaradoss \\ Department of Psychology, \\ Women's Christian College, University of Madras (India) \\ Ebenezer Joseph \\ Principal Investigator, \\ DST Project, Government of India \\ David Chandran \\ Coinvestigator, \\ DST Project, Government of India \\ Sweta Jain \\ Department of Psychology, \\ Ethiraj College for Women, University of Madras (India) \\ Ashwath Paul \\ Department of Statistics, \\ Loyola College, University of Madras (India)
}

\begin{abstract}
Processing speed is one of the measures of cognitive efficiency. It refers to the ability to automatically and fluently execute relatively easy or overlearned cognitive tasks, especially when high mental efficiency is required. Contemporary research has shown that the speed of information processing is dynamically associated with mental capacity, reading performance, and development and reasoning by the conservation of cognitive resources and the efficient use of working memory. While the impact of chess training on intelligence and academic performance has been unequivocally established, its impact on processing speed needs to be researched. This study, funded by the Cognitive Science Research Initiative, Department of Science and Technology, Government of India, analyzed the effect of 1-year chess training on the processing speed of children. A pretest-posttest with control group design was used, with 88 children in the experimental group and 90 children in the control group. The sample was selected from children studying in four city schools (grades 3-9), which included both genders. The experimental group underwent weekly chess training for 1 year, while the control group was involved in extracurricular activities. Processing speed was measured by two subtests of Wechsler Intelligence Scale for Children (WISC-IV India), namely Coding and Symbol Search. The training methodology comprised Winning Moves Chess Learning Program with the demonstration board, on-the-board playing and training, chess exercise through workbooks, and working with chess software. Analysis revealed significant gains in processing speed in the experimental group compared with the control group. The present study clearly establishes a link between chess training and processing speed. Enhancing processing speed in children is especially important because of its relationship to other cognitive and academic performance indicators.
\end{abstract}

Keywords: Chess training, Cognitive development, Schoolchildren, Processing Speed 


\section{INTRODUCTION}

Processing speed relates to an individual's ability to perform simple repetitive cognitive tasks quickly and automatically [1]. It is a cognitive ability that could be defined as the time it takes a person to do a mental task and is related to the speed with which a person can understand and react to the information they receive, whether it be visual (letters and numbers), auditory (language), or movement. In other words, processing speed is the time between receiving and responding to a stimulus.

Processing speed is one of the measures of cognitive efficiency or cognitive proficiency. It involves the ability to automatically and fluently perform relatively easy or overlearned cognitive tasks, especially when high mental efficiency is required. It relates to the ability to process information automatically and therefore speedily, without intentional thinking through. Processing speed is one of the main elements of the cognitive process, which is why it is one of the most important skills in learning, academic performance, intellectual development, reasoning, and experience. Slow processing speed means that some determined tasks will be more difficult than others, like reading, doing math, listening and taking notes, or holding conversations. It may also interfere with executive functions, as a person with slow processing speed will have a harder time planning, setting goals, making decisions, starting tasks, paying attention, etc. The higher the processing speed, the more efficiently you are able to think and learn.

Processing speed could be one of the key processes underlying performance of cognitive tasks under pressure, such as recognizing simple visual patterns, visual exploration tasks, taking tests that require simple decision making, doing basic mathematical calculations, or manipulating numbers. The key to improving processing speed is based on making more solid connections in the brain, which allows the signals to travel faster among each other.

Burns [2] distinguished between two types of mechanisms that may underlie chess skills. Fast mechanisms involve recognition and slow mechanisms refer to a search through the space of positive and negative moves. Speed of processing differentiates these two processes. They concluded that variation in fast processes accounts for substantial variations in chess skills. Chess experts are less affected by time constraints than novices. According to Ericsson and Lehmann [3] processing speed increases with expertise.

Kiesel et al. [4] investigated whether expertise in certain stimulus domain improves perceptual processing to an extent that allows complex visual stimuli to bias behavior unconsciously. Chess experts, but not novice chess players, revealed a subliminal response priming effect, that is, faster responding when prime and target displays were congruent (both checking or both nonchecking) rather than incongruent. The authors conclude that long-term practice prompts the acquisition of visual memories of chess configurations with integrated form-location conjunctions. These perceptual chunks enable complex visual processing outside of conscious awareness.

A number of studies have established that chess learning clearly improves cognitive functioning and academic performance. It is likely that an increase in processing speed is one of the basic factors that supports these gains. However, very few studies appear to have focused on the role of chess training in strengthening processing speed.

Joseph et al. [5] assessed the impact of chess intervention on the IQ scores of children and analyzed the cognitive functions that contributed to the IQ gain. Eighty-six schoolchildren, boys and girls in the age group of 4-15 years, undergoing chess training in an academy, were 
assessed using Binet-Kamat Test of Intelligence. Chess intervention consisted of standardized biweekly training sessions of 2 hours duration over a period of 1 year. Paired $t$-tests and regression analysis were carried out. Significant increases were observed in IQ, and regression analysis indicated that nonverbal reasoning, language, and memory significantly contributed to the dependent variable IQ.

It is hypothesized that systematic chess training would significantly increase processing speed in children. The objective of the study was to assess the impact of weekly chess training on the processing speed of school-going children.

\section{METHODOLOGY}

The research design used for the study was pretest-posttest with control group design [6, 7]. The independent variable was the chess training program and the dependent variable was processing speed of children.

The sample consisted of school-going children with 88 children in the experimental group and 90 children in the control group. The children were selected based on the following selection criteria:

- Children who are studying in schools

- Both genders

- Age range between 6 and 14 years (grades 3 to 9)

The mean age for the experimental group was 10.93 years (SD=2.49) and for control group was 11.15 years $(\mathrm{SD}=2.33)$. The experimental group consisted of 38 girls and 50 boys and the control group consisted of 33 girls and 57 boys.

\section{Tools}

Assessment of processing speed was carried out using the WISC-IV [8] assessment tool. The WISC-IV is an individually administered clinical instrument for assessing cognitive ability of children between the ages from 6 years to 16 years 11 months. The test provided subtest and composite scores that represented intellectual functioning in specific cognitive domains as well as a composite score that represented general intellectual ability. The WISC-IV took $1 \frac{11 / 2}{2}$ to 2 hours to complete. To ensure accurate assessment of the variables, an Indian edition of WISCIV was used. WISC-IV India combined the benefits of the most recent version of the WISC and was adapted to be more relevant for India.

Scores on the coding subtest and the symbol search subtest yielded the processing speed index.

Key features include:

- Culture-appropriate items

- Norms for children in India

- Increased developmental appropriateness

- Increased emphasis on composite scores

- Increased attention to working memory and processing speed

- More user-friendly administration and scoring

- Reflects current theory and practice of cognitive assessment of children

\section{Chess Training Methodology}

The training methodology comprised of Winning Moves Chess Learning Program [9] Episodes 1-22, lectures with the demonstration board, on-the-board playing and training, chess exercise through workbooks (Chess school 1A, Chess school 2, and tactics), and working with chess software. Further students' games were mapped using chess base software and the brain 
patterns of the child were understood. They were taught the ideas behind chess openings and were exposed to classical games. The children participated in mock as well as regular tournaments.

\section{PROCEDURE}

Baseline assessment of processing speed was done after obtaining informed consent. Reassessment was carried after an average duration of 1 year. Psychologists were trained to administer the test in a uniform standardized method to minimize the testing error. Children with an IQ below 80 (WISC-IV India edition) were excluded from the sample.

Clustering technique was used to form the training groups of 6 to 8 children. The chess training consisted of once a week chess classes conducted for 1 hour during school hours at the end of the day. The children were given a standardized Winning Moves Chess Learning Program [9] and played at tournaments also.

\section{RESULTS}

The analysis was carried out using SPSS. Analysis of covariance was carried out to establish the significance of the difference between the experimental and control groups on the processing speed scores following intervention, holding the preintervention scores as covariate.

Table 1: Significance of the difference between the means of the experimental and control groups on processing speed using analysis of covariance

\begin{tabular}{|c|c|c|c|}
\hline \multicolumn{1}{|c|}{ Scores } & Assessment & \multicolumn{2}{|c|}{ Mean and Standard Deviation } \\
\hline \multirow{4}{*}{$\begin{array}{l}\text { Processing } \\
\text { speed }\end{array}$} & \multirow{2}{*}{ Pre } & Experimental & Control \\
\cline { 2 - 3 } & \multirow{3}{*}{ Post } & $\begin{array}{c}96.705 \\
(13.592)\end{array}$ & $\begin{array}{c}96.200 \\
(14.372)\end{array}$ \\
\cline { 2 - 4 } & & $\begin{array}{c}103.841 \\
(13.550)\end{array}$ & $\begin{array}{c}99.811 \\
(14.430)\end{array}$ \\
\hline
\end{tabular}

ANCOVA Between Experimental Group and Control Group on Processing Speed at Postintervention

\begin{tabular}{|c|c|c|c|c|c|}
\hline Sources of Variance & $\begin{array}{l}\text { Sum of } \\
\text { Squares }\end{array}$ & $d f$ & $\begin{array}{c}\text { Mean } \\
\text { Square }\end{array}$ & $F$ & Sig. \\
\hline Corrected model & $16,098.206^{\mathrm{a}}$ & 2 & $8,049.103$ & 73.633 & 0.000 \\
\hline Intercept & $5,073.88$ & 1 & $5,073.88$ & 46.416 & 0.000 \\
\hline Preprocessing speed & $15,375.65$ & 1 & $15,375.65$ & 140.656 & 0.000 \\
\hline EXPCON & 606.547 & 1 & 606.547 & 5.549 & 0.02 \\
\hline Error & $19,129.91$ & 175 & 109.314 & & \\
\hline Total & $1,880,007$ & 178 & & & \\
\hline Corrected total & $35,228.12$ & 177 & & & \\
\hline
\end{tabular}

Table 1 indicates that there was a significant effect of intervention on processing speed at postintervention $(p<0.02)$. This shows that the mean processing speed increased in the experimental group following intervention, compared with the control group.

\section{DISCUSSION}

The results clearly demonstrate that chess training has increased the processing speed of the children who underwent chess training when compared with the control group. Processing 
speed has been assessed using the Coding subtest (the child copies the symbol that is paired with geometric shapes or numbers using a key within a specified time limit) and the Symbol Search subtest (where the child scans a search group and indicates whether the target symbol(s) matches any of the symbols in the search group within a specified time limit).

Earlier studies have reported that chess playing has resulted in increased working memory and perceptual reasoning skills $[10,11]$. It is possible that enhancement of these cognitive functions could have possibly led to an increase in the processing speed of children. A clear link between working memory, processing speed, and reasoning has been established by clinical research in developmental cognitive neuropsychology [12].

Alternatively, it is possible that chess intervention directly influences the processing speed ability. Chess playing includes two methods of processing information and decision making on every single move. They are either by judgment or by calculation. Certain position requires just the positional knowledge to make a move. For instance, which side to castle, where to attack, where the point of action is, etc. Certain tactical positions require detailed calculations of the various options and outcomes that could possibly evolve out of each candidate move. A candidate move is one of the moves that the chess player thinks to be analyzed in detail at any given position using the tree method of thinking.

Burns [2] emphasized that variation in fast processes accounts for substantial variations in chess skills. According to Ericsson and Lehmann [3], processing speed increases with expertise. De Groot et al. [13] highlighted the chess master's striking ability to quickly capture, and to retain and recall, the information contained in an unknown, complex chess position: its gist and its structure, up to the precise location of almost all chess pieces. This speed in understanding a position is obviously a basic element of the master's expertise and characterizes one's way of thinking in general. Increased processing speed scores (as measured by the coding and symbol search subtest) could in addition reflect increases in short-term memory, visual scanning ability, visual and sequential processing, visual-motor coordination, cognitive flexibility, visual discrimination, and concentration and planning and learning ability, following intervention $[14,15]$.

Processing speed has been identified as an important domain of cognitive functioning in factor analytical studies of cognitive abilities [16]. It may be important to both assess and sharpen the processing speed in children because of its relation to neurological development, other cognitive abilities, and learning. Improvements in children's performance on measures of processing speed are mirrored by age-related changes in the number of transient connections in the nervous system and increase in myelination [17].

Identifying increases in processing speed following chess intervention provides us with a framework to appreciate the impact of chess on the broader, global capacities that have been extensively researched. Processing speed is a critical ingredient in optimal cognitive functioning.

\section{IMPLICATIONS}

The role played by chess training in increasing processing speed has been clearly established in this study. This is a significant finding as processing speed ability contributes significantly by conserving cognitive resources and efficiently using the working memory for higher order fluid tasks. The link between chess and processing speed needs to be further researched in order to clearly understand its potential benefits and its implications in education. 


\section{References}

Schneider, W. J., \& McGrew, K. S. (2012). The Cattell-Horn-Carroll model of intelligence. In D. P. Flanagan \& P. L. Harrison (Eds.), Contemporary intellectual assessment: Theories, tests, and issues (3rd ed., pp. 99-144). New York, NY: Guilford Press.

Burns, B. D. (2004).The effects of speed on skilled chess performance. Psychological Science, 15(7), 442-447.

Ericsson, K. A., \& Lehmann, A. C. (1996). Expert and exceptional performance: Evidence on maximal adaptations on task constraints. Annual Review of Psychology, 47, 273-305.

Kiesel, A., Kunde, W., Pohl, C., Berner, M., \& Hoffmann, J. (2009). Playing chess unconsciously. Journal of Experimental Psychology: Learning, Memory, and Cognition, 35, 292-298.

Joseph, E., Easvaradoss, V., \& Solomon, N. J. (2016). Impact of chess training on academic performance of rural Indian school children. Open Journal of Social Sciences, 4, 20-24. Retrieved from http://dx.doi.org/10.4236/jss.2016.42004

Edwards, A. L. (1985). Experimental designs in psychological research. New York, NY: Harper \& Row.

Kerlinger, F. N. (1973). Foundation of behavioural research. New York, NY: Holt, Rinehart and Winston.

Wechsler, D., Kaplan, E., Fein, D., Kramer, J., Morris, R., Delis, D., \& Maelender, A. (2003). Wechsler intelligence scale for children: Fourth edition (WISC-IV) [Assessment instrument] (Indian adaptation). San Antonio, TX: Pearson.

Joseph, E. (2008). Winning Moves Chess Learning Program, Patent No. L-32958/2009, India.

Easvaradoss, V., Joseph, E., Chandrasekaran, S., Jain, S., Mathai, A., \& Christy, S. (2017). Improving working memory in school children through chess training. International Journal of Psychological and Behavioral Sciences, 11(5), 5 .

Joseph, E., Easvaradoss, V., Manoharan, S. S., Chandran, D., Chandrasekaran, S., \& Uma, T. R. (2017). Improving perceptual reasoning in school children through chess training. International Journal of Psychological and Behavioral Sciences, 11(5), 90.

Fry, A. F., \& Hale, S. (1996). Processing speed, working memory and fluid intelligence: Evidence for a developmental cascade. Psychological Science, 7(4), 231-241.

de Groot, A. D., Gobet, F., \& Jongman, R. W. (1996). Perception and memory in chess: Studies in the heuristics of the professional eye. Assen, the Netherlands: Van Gircum.

Kaufman, A. S. (1994). Intelligent testing with the WISC III. New York, NY: Wiley.

Sattler, J. M. (2001). Assessment of children: Cognitive applications (4th ed.). San Diego, CA: Author.

Horn, J. L., \& Noll, J. (1997). Human cognitive capabilities: Gf-Gc theory. In D. P. Flangan, J. L. Genshaft, \& P. L. Harrison (Eds.), Contemporary intellectual assessment: Theories, tests and issues (pp. 53-91). New York, NY: Guilford Press.

Cepeda, N. J., Kramer, A. F., \& Gonzalezde Sather, J. C. M. (2001). Changes in executive control across the life span: Examination of task switching performance. Developmental Psychology, 37(5), 715-730. 